Notfall Rettungsmed 2013 · 16:8-9

DOI 10.1007/s10049-012-1672-8

Online publiziert: 1. Februar 2013

(c) Springer-Verlag Berlin Heidelberg 2013

\author{
H.-R. Arntz ${ }^{1}$ U. Zeymer ${ }^{2}$ \\ ${ }^{1}$ Medizinische Klinik II, Kardiologie-Pulmologie, Charité - \\ Universitätsmedizin Berlin, Campus Benjamin Franklin \\ ${ }^{2}$ Medizinische Klinik B, Klinikum Ludwigshafen
}

\title{
Akuter Herzinfarkt
}

\author{
Neue Definitionen, Netzwerke \\ zur Therapie und die Beziehung \\ zum plötzlichen Herztod
}

Der akute Myokardinfarkt konventioneller Definition (ST-Streckenhebungsinfarkt) nimmt in seiner Häufigkeit seit Jahren ab, nicht jedoch die Häufigkeit anderer ischämischer myokardialer Ereignisse, die mit Zelluntergang (Myokardnekrosen) einhergehen. In der Tat zeigen letztere Ereignisse eher eine Zunahme. Erklärbar ist dies sicher z.T. durch die im Laufe der Jahre erheblich verbesserte primäre und sekundäre Prävention einschließlich wirksamerer invasiver Behandlungsverfahren der koronaren Herzerkrankung (KHK). Die Änderungen des Erscheinungsbilds der Akutmanifestation der KHK haben nicht nur therapeutische, sondern auch diagnostische Konsequenzen und verlangen präzisere Definitionen dessen, was bisher unter dem pauschalisierenden Begriff „Infarkt“ zusammengefasst wurde. Ein Beispiel soll dies verdeutlichen: Im Rahmen von Koronarinterventionen sowohl per Kathetertechnik als auch bei koronarchirurgischen Interventionen kann es unter unglücklichen Umständen zu erheblichen myokardialen Zelluntergängen kommen. Schon aus Sicht der Einordnung dieser Ereignisse für die Prognose der Patienten und die Therapiewahl bedarf es einer eigenen definierten Kategorie für diese Besonderheit. Eine Arbeit im Leitthema dieser Ausgabe von Notfall und Rettungsmedizin beschäftigt sich mit den aktuellen Definitionen und dem seit neuerem verfügbaren diagnostischen Instrumentarium sowie den daraus folgenden Konsequenzen für die Notfallversorgung.

In einem weiteren Beitrag wird das Zentralproblem der Versorgung von $\mathrm{Pa}$ tienten mit akutem ST-Streckenhebungsinfarkt (STEMI) angegangen, nämlich der Zeitverlust zwischen Beginn der Ischämiesymptomatik und der effektiven Reperfusionstherapie. Während die Verkürzung der Reaktionszeit der Patienten bis zum Hilfeersuchen ein sehr komplexes bisher auch nicht befriedigend gelöstes Problem darstellt, gibt es für die Zeitfenster zwischen erstem medizinischem Kontakt, der nach Möglichkeit natürlich über den Rettungsdienst erfolgen sollte, und definitiver Reperfusionsbehandlung ebenso klare Vorgaben wie für die notwendigen ersten diagnostischen Schritte und die Auswahl der Therapiestrategie primärer kathetertechnischer Koronarintervention (PPCI) bzw. Lysetherapie. Um die genannten Zeitfenster so klein wie möglich zu halten bzw. im Rahmen der Vorgaben der Leitlinien zu halten und gleichzeitig der bevorzugten Therapiestrategie der PPCI den Weg zu ebnen, haben sich Netzwerke aus Rettungsdienst sowie Kliniken mit und ohne Möglichkeit der 24-stündigen täglich verfügbaren PCI-Bereitschaft bewährt. In der vorliegenden Arbeit werden die akzeptablen Zeitfenster diskutiert, die zeitlichen Anforderungen an ein solches Netzwerk, Wege zur seiner Etablierung und therapeutische Alternativen mit dem Ziel, möglichst vielen Patienten eine optimale und frühzeitige Reperfusionstherapie anzubieten.

\section{》) Der plötzliche Herztod ist bei weitem nicht so plötzlich wie es der Begriff suggeriert}

Eine letzte Arbeit beschäftigt sich mit der Katastrophe bei der Manifestation der koronaren Herzkrankheit, dem plötzlichen Herztod. Wie sich herausstellt ist der plötzliche Herztod bei weitem nicht so plötzlich wie es der Begriff suggeriert. Er steht in engem Zusammenhang mit einer oft dem Opfer schon bekannten chronischen KHK bzw. tritt als Komplikation einer ersten Akutmanifestation der KHK ein. Ähnlich wie auch beim zunächst unkomplizierten Myokardinfarkt bestehen die typischen ischämischen Beschwerden oft lange gelegentlich über Stunden, bevor es schließlich zum Kollaps bei Kreislaufstillstand kommt. Während im ersteren Falle das Ausmaß der myokardialen Schädigung v. a. die Lebensqualität, aber auch die Lebenserwartung bestimmt, ist im zweiten Fall das Zeitfenster für eine Rettung auf wenige Minuten beschränkt und besonders das Überleben von der richtigen Reaktion und richtigen Ersthelfermaßnahmen von Augenzeugen abhängig. Leider zeigt sich, dass insbesondere in häuslicher Umgebung trotz häufiger Gegenwart von Augenzeugen weder 
angemessen auf die Symptomatik reagiert wird noch bei Eintritt des Kreislaufstillstands zu den richtigen Maßnahmen gegriffen wird - ein reiches Betätigungsfeld für Aufklärung und Schulung.

Wir hoffen, mit dieser kleinen Leitthemenserie auf Ihr Interesse zu stoßen und Ihnen einige Anregungen für Ihre praktische tägliche Arbeit gegeben zu haben.

Ihre

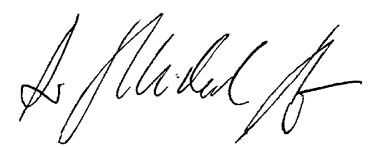

Hans-Richard Arntz

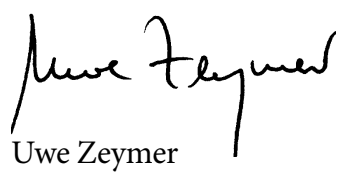

\section{Korrespondenzadresse}

\section{Prof. Dr. H.-R. Arntz}

Medizinische Klinik II, Kardiologie-Pulmologie, Charité - Universitätsmedizin Berlin, Campus Benjamin Franklin Hindenburgdamm 30, 12200 Berlin hans-richard.arntz@charite.de

\section{Compliance with Ethics Guidelines}

Conflict of Interest. U. Kreimeier and H.R. Arntz declare that they have no conflict of interest.

This article does not contain any studies with human or animal subjects.

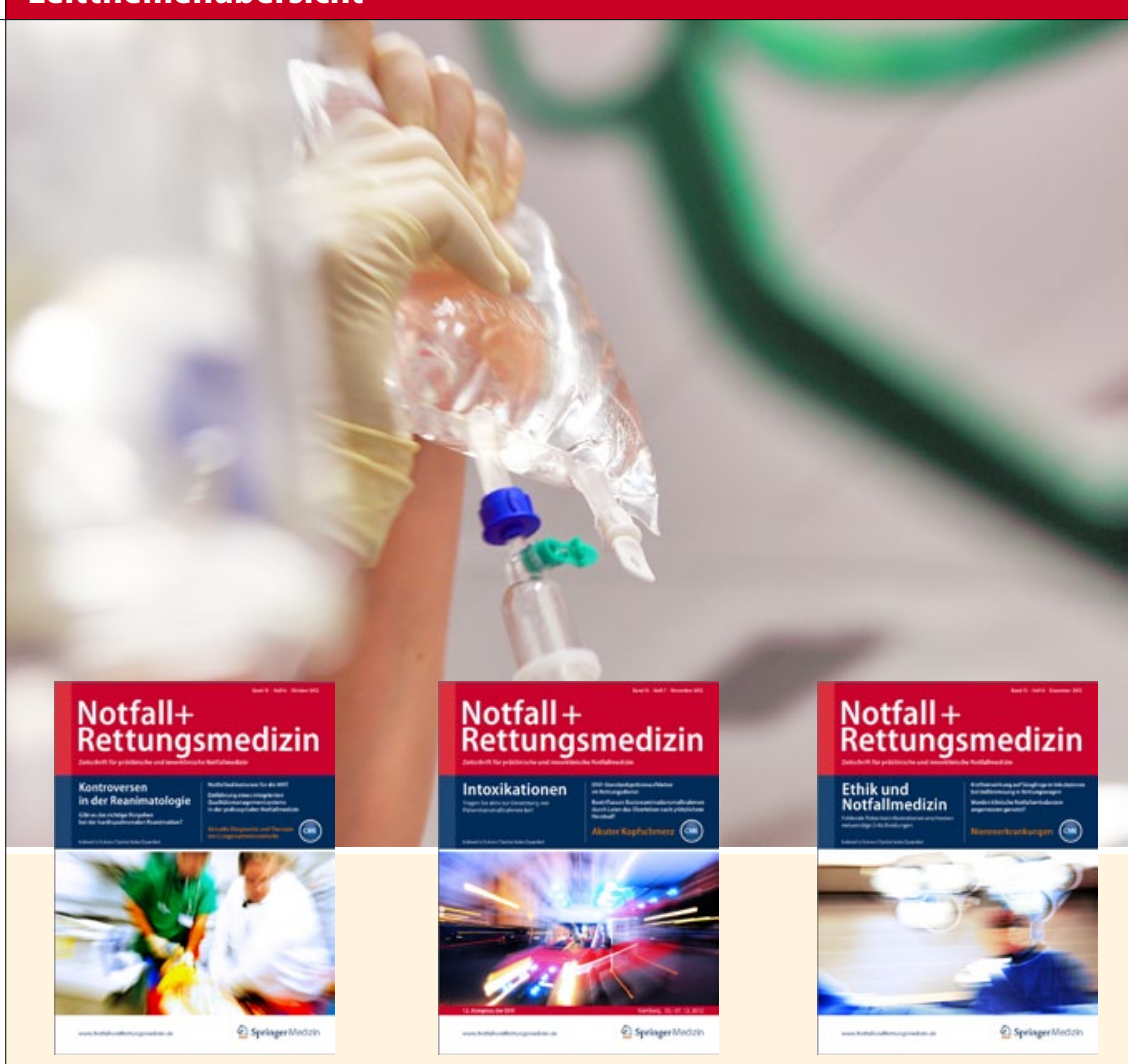

Die Notfall + Rettungsmedizin bietet Ihnen jeden Monat umfassende und aktuelle Beiträge zu interessanten Themenschwerpunkten aus allen Bereichen der Notfallmedizin. Möchten Sie ein bereits erschienenes Heft nachbestellen? Einzelne Ausgaben können Sie direkt bei unserem Kundenservice zum Preis von je EUR 35,- zzgl. Versandkosten beziehen:

\section{Überblick 2011}

1/11 Präklinisches Atemwegsmanagement

2/11 Lebensgefährliche Herzrhythmusstörungen

3/11 Notfallmedizin und Informationstechnologie

4/11 Leichte Schädel-Hirn-Traumata

$5 / 11$ Notaufnahme

6/11 Medizinrechtliche Aspekte der Notfallmedizin

7/11 Pädiatrische Notfälle

8/11 Advanced Technology in der Notfallmedizin

\section{Überblick 2012}

1/12 Risikomanagement in der Akutmedizin

2/12 Respiratiorsche Notfälle im Kindesalter

3/12 Weiterbildung in der Notfallmedizin

4/12 Schnittstellen in der Notfallmedizin

5/12 Notaufnahme: Arbeitsplatz mit Zukunft?

6/12 Kontroversen in der Reanimatologie

$7 / 12$ Intoxikationen

8/12 Ethik in der Notfallmedizin

So erreichen Sie unseren Kundenservice:

Springer Customer Service Center GmbH Kundenservice Zeitschriften

Haberstr. 7

69126 Heidelberg

Tel.: +49 $6221345-4303$

Fax: +49 $6221345-4229$

E-Mail: leserservice@springer.com

\section{www.NotfallundRettungsmedizin.de}

